Article

\title{
The Characteristics of the Growth and the Active Compounds of Angelica gigas Nakai in Cultivation Sites
}

\author{
Yunmi Park ${ }^{1, *}$, Pil Sun Park ${ }^{2}(0)$, Dae Hui Jeong ${ }^{3}$, Sujin Sim ${ }^{4}$, Nahyun Kim ${ }^{3}$, Hongwoo Park ${ }^{3}$, \\ Kwon Seok Jeon ${ }^{3}$, Yurry Um ${ }^{3}$ and Mahn-Jo Kim ${ }^{1}$ \\ 1 Department of Forest Bioresources, National Institute of Forest Science, Suwon-si 16631, Korea; \\ otttr@korea.kr \\ 2 Department of Forest Sciences, Seoul National University, Seoul 08826, Korea; pspark@snu.ac.kr \\ 3 Forest Medicinal Research Center, National Institute of Forest Science, Yeongju-si 36040, Korea; \\ najdhda@korea.kr (D.H.J.); knh1125@korea.kr (N.K.); redrain39@korea.kr (H.P.); jks2029@korea.kr (K.S.J.); \\ urspower@korea.kr (Y.U.) \\ 4 Forest Biomaterials Research Center, National Institute of Forest Science, Jinju-si 52817, Korea; \\ sujin0606@korea.kr \\ * Correspondence: pym5250@korea.kr
}

Received: 9 June 2020; Accepted: 27 June 2020; Published: 30 June 2020

check for updates

\begin{abstract}
The active compounds of medicinal plants vary in composition and content depending on environmental factors, such as light, temperature, and soil. According to the Korean Pharmacopoeia standards for herbal medicine, the sum of nodakenin, decursin, and decursinolangelate, which are the marker components of Korean Angelica, should be at least $6.0 \mathrm{~g} / 100 \mathrm{~g}$. However, the content of the components in Korean Angelica cultivated in South Korea often fall below $6.0 \mathrm{~g} / 100 \mathrm{~g}$, due to weather conditions and cultivation site characteristics. This study aimed to gather information about environmental factors that affect the root growth and the content of active compounds. In total, 18 cultivation sites in Pyeongchang, Jecheon, and Bonghwa regions in Korea were investigated for this study. Environmental factors, such as the monthly mean temperature, mean relative humidity, duration of sunshine, total precipitation, soil acidity, and the characteristics of soil nutrient, were investigated over the growing season from April to October 2017. As for the growth characteristics, the dry weight of roots of Korean Angelica was measured. The sum of the contents of the three active compounds was 5.3-7.0 g/100 $\mathrm{g}$ and the nodakenin content was 0.3-1.3 g/100 $\mathrm{g}$ in the cultivation sites. This study concludes that the root yields in the cultivation sites would be improved if weather conditions are maintained with similar levels as those in their natural habitats. Additionally, the environment that improves root growth did not increase the content of active compounds; however, when there was a lot of gravel or high temperatures during the growth period, the content of active compounds was relatively high.
\end{abstract}

Keywords: Angelica gigas Nakai; active compounds; correlation analysis; growth; root 


\section{Introduction}

'Korean Angelica' (Angelica gigas Nakai) is a perennial plant of the genus Umbelliferae, where young shoots are eaten as seasoned vegetables and roots are used for medicinal purposes. The plant grows to a height of 1 to 2 meters, purple double umbrella-shaped flowers bloom between August and September, and there will be many small rootlets on the thick roots [1]. The origin of the Angelica gigas is in East Asia and has been distributed in Korea and northeastern China. However, the confirmed natural habitat on the Korean Peninsula is the cool-temperate mountain areas, which corresponds to mid-to-north highlands and is located in areas adjacent to the valley at 700 to $1300 \mathrm{~m}$ [2]. As Korean Angelica prefers low-temperature and long-day conditions, the roots are wooded, and the medicinal properties are reduced when the summer depression occurs during high temperatures or when the flower stalks are raised early [3]. Due to the physiological and ecological characteristics available in the highlands, the Korean Angelica has been growing in Gangwon Province or in the mountains of North Gyeongsang Province in Korea.

The medicinal properties used by humans are a combination of species-specific secondary metabolites in plants [4]. Unlike primary metabolites, such as carbohydrates, proteins, lipids, chlorophyll, and nucleic acids, which are essential for plants to maintain and increase their cell number, secondary metabolites play an important role in the physiological function of plants. These secondary metabolites not only play an important role in plant chemical defense of species against herbivores [4,5] but also engage in non-biological stresses, such as temperature, moisture, light, and changes in inorganic nutrients. Recently, it has been found to be involved in the regulation of gene expression at the cell level, or signal transmission [6]. Currently, about 300,000 species of plants worldwide are known to produce secondary metabolites. About 1500 types of substances are extracted and separated from plant bodies every year, and about 300 of them are considered as useful materials for biological activity. Most of the plant-generated secondary metabolites are also used to make dyes, polymers, fibers, adhesives, oils, waxes, spices, and perfumes and are useful and valuable in developing new medicines, antibiotics, pesticides, and herbicides [6].

Although 'Korean Angelica' is mentioned more than 500 times in 'Donguibogam' prescriptions and more than 150 times in 'Bangyakhappyeon', the plants used in China and Japan are different from the original plant used in Korea. In Korea, the 'Korean Angelica' (Angelica gigas Nakai) is used, while the 'China Angelica' (Angelica Sinensis Oliv. Diels) is used in China, and the 'Japan Angelica' (Angelica acutilova) is used in Japan [7]. Only the 'Korean Angelica' and 'Japan Angelica' are grown in Korea, as the climate is not suitable for the growth of 'China Angelica' [8]. It has been argued that the Korean Angelica should be used to distinguish its origin in Korea, China, and Japan because it is of great importance in clinical trials. The main active compounds of the Chinese Angelicia are essential oil, organic acids, and polysaccharides, and the major active compounds are z-ligustilide (3-butylylide4,5-dihydrophthalide), which is a neutral oil, butylidenephthalide, and ferulic acid [9]. The Japanese Angelica are generally similar to Chinese Angelica, and contain z-ligustilide, n-butylidenephthalide, ferulic acid, and polysaccharide [10,11], but additionally to these, it contains 9,12-octaneacid [12]. The main active compounds of Korean Angelica are fat-soluble coumarins, which include decursin, decursinolangelate, umberliferone, nodakenin, peucedanone, marmesin, demethylsuberosin, isoimperatorin etc. Among the compounds, decursin, decursinolangelate, and nodakenin are the major active compounds. Decursin is a natural component of the pyranocoumarin system, which was first separated from the ether extract of Angelica decursiva Fr. et Sav. in Japan and has the molecular formula of $\mathrm{C}_{19} \mathrm{H}_{20} \mathrm{O}_{5}$ with a molecular mass of $328.35 \mathrm{~g}$. The Korean Angelica, in particular, contains a large amount of decursin compared with other Angelicas [13].

The most active areas of research on the pharmacology of Angelica gigas Nakai, Angelica Sinensis Oliv. Diels, and the Angelica acutilova are on their effects on decreasing the blood pressure, ischemic heart disease treatment, blood clotting delay [14-16] effects on the central nervous system, protection against neurotoxicity, and improvement of memory $[17,18]$. The study of anticancer action is mainly based on studies of decursin and decursinolangelate, which are the main ingredients of Korean Angelica [19]. 
In addition, continuous research has been conducted on areas, such as immunity, anti-inflammatory, and hemorrhage properties $[15,20]$. Nodakenin is likely to be used as a medicine and health function food for the prevention and treatment of degenerative brain diseases due to its nerve cell protection properties [15]. Nodakenin also inhibits the secretion of allergens in fat cells and showed excellent anti-allergic effects in allergy animal models [15,16]. Most of the Korean Angelica cultivation sites today are created by planting seeds taken from their natural habitat; however, these cultivation sites often do not contain optimum conditions needed for the production of increased levels of roots and active compounds. Apart from these adverse conditions, due to the changes in weather conditions also, the amounts of active compounds, which are secondary metabolites, are less than $6 \mathrm{~g} / 100 \mathrm{~g}$ as stipulated by the Korean Pharmacopoeia. It is predicted that the number of Korean Angelica cultivation sites, which are vulnerable to climate change, will decrease from $12 \%$ of the total current cultivation areas to less than 1\% by 2040 [21]. The active compounds of medicinal plants vary in composition and content depending on the period of growth and the environment, such as light, temperature, and soil. Korean Angelica has been cultivated in sites in various locations and environmental conditions, and its supply and demand as raw material is not smooth due to the fluctuating content of active compounds depending on environmental factors.

Therefore, research on environmental factors that affect the content of active compounds is needed to improve the quality of locally produced Korean Angelica as a functional raw material. Until now, no studies have been conducted in which the active compounds of the Korean Angelica were analyzed together with the characteristics of the growth in the cultivation site $[7,13]$. Thus, this study aimed to identify the environmental factors affecting the root growth and content of active compounds in the roots of Korean Angelicas grown in the cultivation sites and to establish a database to select optimal cultivation conditions.

\section{Results}

\subsection{Soil Characteristics}

An analysis of the gravel content of the soil in the cultivation sites showed that the content did not show much difference between the cultivation sites in Pyeongchang, and varied between $30.0 \%$ and $38.9 \%$. On the other hand, all of the sites in Jecheon showed a high value of over 35\% on average, with a large difference in the amount of gravel among the sites. In particular, Je1-3 had the highest amount among the 15 sites with a $62.0 \%$ gravel content. In the Bonghwa, Bon- 2 had the highest gravel content at $44.4 \%$ while Bon-3 had the lowest at $26.0 \%$. Among the 18 sites in all three regions, Pye $1-1$ had the highest statistically significant clay content, which was at $14.5 \%$, while the Bon-3 and Bon- 4 sites had the lowest clay content at $5.0 \%$ to $6.4 \%$ (Table $1, p<0.05$ ).

The chemical properties of soil were generally high in the order of Pyeongchang, Jecheon, and Bonghwa by region, but they showed various characteristics for each site within the region. In the case of soil acidity, out of a total of 18 cultivation sites, the highest was seen in Je4-2 at $7.7 \mathrm{pH}$ and the lowest was seen in Pye2-5 at $4.2 \mathrm{pH}$. The content of organic matter, available $\mathrm{P}_{2} \mathrm{O}_{5}$, and the cation exchange capacity (CEC), which are indicators of soil nutrients, were statistically significantly higher in Pyeongchang than in other regions. In the case of cation exchange, out of a total of 18 cultivation sites, the highest $\mathrm{K}^{+}$was seen in Je2-1 at $1.0 \mathrm{cmolc} \mathrm{kg}^{-1}$. The highest $\mathrm{Na}^{+}$was in Je1-3 and the lowest $\mathrm{Ca}^{2+}$ was seen in Pye2-5. The lowest $\mathrm{Mg}^{2+}$ was in Pye2-1 at $0.3 \mathrm{cmolc} \mathrm{kg}^{-1}$ and the highest in Je3-2 at 2.4 cmolc $\mathrm{kg}^{-1}$ (Table 2, $p<0.05$ ). 
Table 1. Soil textures in Angelica gigas cultivation sites, Korea (mean \pm SD).

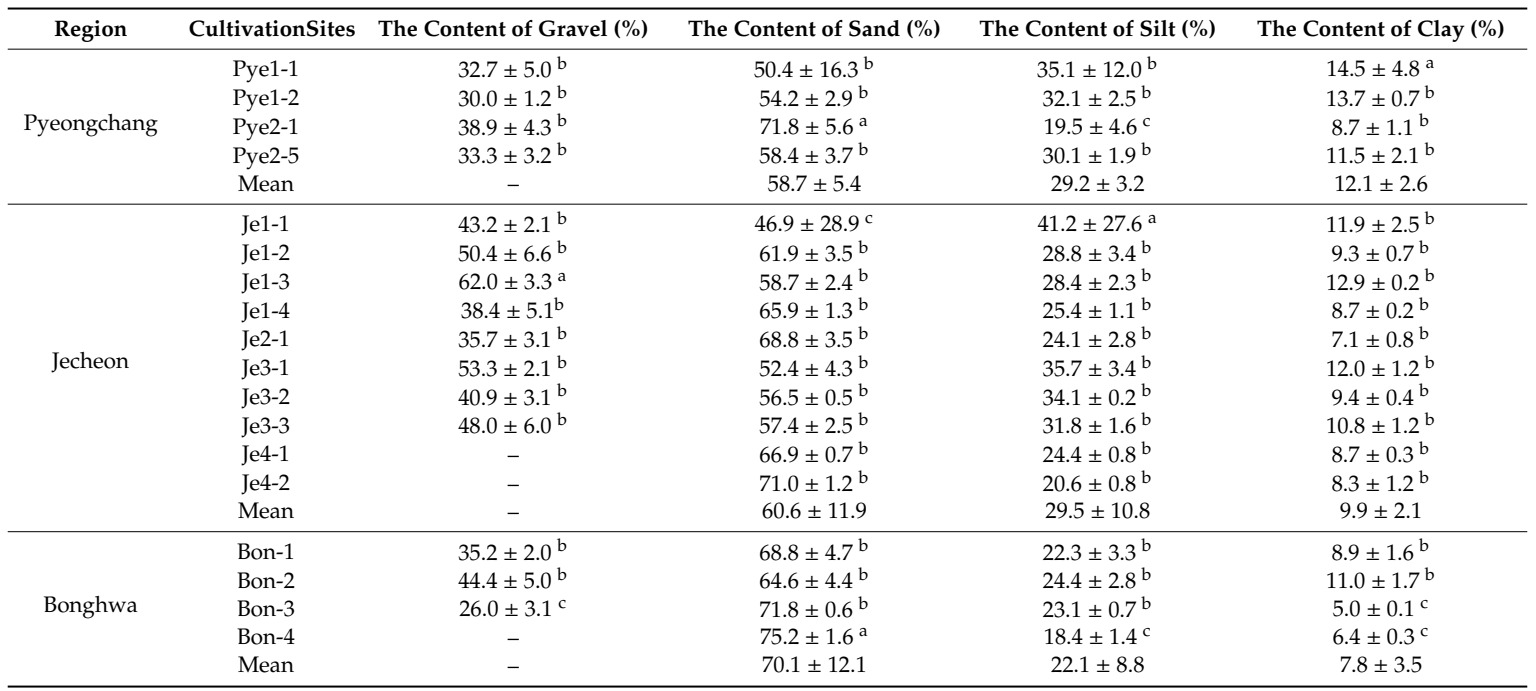

${ }^{*} \mathrm{a}, \mathrm{b}, \mathrm{c}$ : value in same columns with different superscripts are significantly different $(p<0.05)$ with one way ANOVA and Duncan's test (alpha =0.05) using SPSS statistics (IBM Corporation, Ver. 20.0 Armonk, NY, USA).

\subsection{Growth Characteristics}

The Pyeongchang region showed the highest average fresh weight of individual roots, which was $467.5 \pm 275.6 \mathrm{~g}$, while Jecheon area showed the lowest value at $293.9 \pm 218.6 \mathrm{~g}$. The root dry weight was 100.4 to $167.9 \mathrm{~g}$ in the Pyeongchang area, while in the Jecheon area, an average value of less than $100 \mathrm{~g}$ was shown, with a wide variation of 33.5 to $149.4 \mathrm{~g}$ between the cultivation sites. According to the characteristics of the cultivation sites, the fresh weight and dry weight of the roots in the Pye1-2 were statistically the highest among the 18 sites in the entire region. In addition, Pye2-5 has the highest fresh weight of a root/shoot growth ratio of 5.3, making the root growth far superior to that of the shoot growth. Je1-1 and Bon-4 both showed statistically the lowest root/shoot ratio among the 18 cultivation sites (Table $3, p<0.05$ ).

Correlation analysis was performed between the root dry weight, water content in root, and root/shoot ratio, which are the main growth characteristics for the cultivation sites, and data obtained from the weather measuring devices installed in each site. As a result, the root dry weight showed a negative correlation with temperature-related factors from April to October, and a positive correlation with altitude and air humidity (Table 4 , significant at $p=0.01$ ). Additionally, the root/shoot ratio showed a positive correlation with altitude (Table 4 , significant at $p=0.01$ ). An analysis of the correlation between the soil characteristics of the cultivation sites and root growth characteristics showed that root dry weight showed a positive correlation with the organic matter content (Table 5, significant at $p=0.01$ ). 
Table 2. Edaphic characteristics of Angelica gigas cultivation sites, Korea.

\begin{tabular}{|c|c|c|c|c|c|c|c|c|c|c|}
\hline Region & $\begin{array}{l}\text { Cultivated } \\
\text { Sites }\end{array}$ & $\begin{array}{c}\mathrm{pH} \\
(1: 5, \mathrm{H} 2 \mathrm{O})\end{array}$ & $\begin{array}{c}\text { Organic Matter } \\
(\mathrm{g} / \mathbf{1 0 0 g})\end{array}$ & $\begin{array}{l}\text { Total N } \\
(\mathrm{g} / \mathbf{1 0 0 g})\end{array}$ & $\begin{array}{c}\text { Available } \mathrm{P}_{2} \mathrm{O}_{5} \\
(\mathrm{mg} / \mathrm{kg})\end{array}$ & $\begin{array}{c}\text { CEC } \\
\left(\mathrm{cmolc} \mathrm{kg}^{-1}\right)\end{array}$ & $\mathrm{K}^{+}$ & $\mathrm{Na}^{+}$ & $\mathrm{Ca}^{2+}$ & $\mathrm{Mg}^{2+}$ \\
\hline & & & & & & & & & \multicolumn{2}{|c|}{ cmolc kg $^{-1}$} \\
\hline \multirow{5}{*}{ Pyeongchang } & Pye1-1 & $4.6 \pm 0.2^{c}$ & $6.4 \pm 2.9^{a}$ & $0.4 \pm 0.1^{\mathrm{a}}$ & $990 \pm 38^{b}$ & $13.8 \pm 4.2^{b}$ & $0.9 \pm 0.3^{b}$ & $0.1 \pm 0.0^{b}$ & $4.1 \pm 0.9^{b}$ & $0.8 \pm 0.2^{b}$ \\
\hline & Pye1-2 & $5.0 \pm 0.2^{b}$ & $6.9 \pm 0.3^{a}$ & $0.4 \pm 0.0^{a}$ & $1121 \pm 124^{b}$ & $14.8 \pm 0.6^{\mathrm{a}}$ & $0.9 \pm 0.2^{b}$ & $0.1 \pm 0.0^{b}$ & $4.1 \pm 0.6^{b}$ & $0.6 \pm 0.1^{b}$ \\
\hline & Pye2-1 & $4.4 \pm 0.6^{b}$ & $3.9 \pm 0.8^{b}$ & $0.3 \pm 0.1^{b}$ & $1456 \pm 57^{a}$ & $10.3 \pm 0.8^{b}$ & $0.7 \pm 0.1^{b}$ & $0.1 \pm 0.0^{b}$ & $2.7 \pm 0.2^{b}$ & $0.3 \pm 0.1^{\mathrm{c}}$ \\
\hline & Pye2-5 & $4.2 \pm 0.2^{c}$ & $6.0 \pm 0.7^{\mathrm{a}}$ & $0.4 \pm 0.0^{b}$ & $1018 \pm 235^{b}$ & $12.9 \pm 1.0^{\mathrm{a}}$ & $0.6 \pm 0.1^{b}$ & $0.1 \pm 0.0^{b}$ & $2.3 \pm 0.5^{c}$ & $0.4 \pm 0.2^{b}$ \\
\hline & Mean & $4.6 \pm 0.5$ & $5.8 \pm 1.9$ & $0.4 \pm 0.1$ & $1146 \pm 231$ & $13.0 \pm 2.8$ & $0.8 \pm 0.2$ & $0.1 \pm 0.0$ & $3.3 \pm 1.0$ & $0.5 \pm 0.2$ \\
\hline \multirow{11}{*}{ Jecheon } & Je1-1 & $5.0 \pm 0.2^{b}$ & $1.0 \pm 0.2^{b}$ & $0.1 \pm 0.0^{b}$ & $423 \pm 213^{b}$ & $8.1 \pm 0.9^{b}$ & $0.3 \pm 0.0^{b}$ & $0.1 \pm 0.0^{b}$ & $2.3 \pm 0.5^{c}$ & $0.7 \pm 0.2^{b}$ \\
\hline & Je1-2 & $5.9 \pm 0.1^{b}$ & $3.6 \pm 0.4^{b}$ & $0.3 \pm 0.0^{b}$ & $977 \pm 30^{b}$ & $11.9 \pm 0.2^{b}$ & $0.7 \pm 0.2^{b}$ & $0.1 \pm 0.0^{b}$ & $4.7 \pm 0.5^{b}$ & $0.7 \pm 0.0^{b}$ \\
\hline & Je1-3 & $6.6 \pm 0.5^{b}$ & $2.3 \pm 0.3^{b}$ & $0.2 \pm 0.0^{b}$ & $419 \pm 34^{c}$ & $10.0 \pm 0.5^{b}$ & $0.4 \pm 0.1^{\mathrm{b}}$ & $0.1 \pm 0.0^{\mathrm{a}}$ & $5.5 \pm 0.9^{b}$ & $0.4 \pm 0.1^{b}$ \\
\hline & Je1-4 & $6.3 \pm 0.7^{b}$ & $2.5 \pm 0.7^{b}$ & $0.2 \pm 0.0^{b}$ & $670 \pm 115^{b}$ & $9.0 \pm 1.2^{b}$ & $0.5 \pm 0.1^{b}$ & $0.1 \pm 0.0^{b}$ & $4.6 \pm 1.6^{\mathrm{b}}$ & $0.4 \pm 0.2^{b}$ \\
\hline & Je2-1 & $6.3 \pm 0.2^{b}$ & $2.6 \pm 0.3^{b}$ & $0.2 \pm 0.0^{\mathrm{b}}$ & $991 \pm 56^{\mathrm{b}}$ & $10.9 \pm 0.5^{b}$ & $1.0 \pm 0.1^{\mathrm{a}}$ & $0.1 \pm 0.0^{b}$ & $4.6 \pm 0.2^{b}$ & $0.9 \pm 0.1^{b}$ \\
\hline & Je3-1 & $5.5 \pm 0.4^{b}$ & $3.7 \pm 0.8^{b}$ & $0.3 \pm 0.0^{b}$ & $953 \pm 83^{b}$ & $12.3 \pm 1.5^{\mathrm{a}}$ & $0.8 \pm 0.1^{b}$ & $0.1 \pm 0.0^{b}$ & $3.3 \pm 0.8^{b}$ & $1.1 \pm 0.3^{b}$ \\
\hline & Je3-2 & $6.4 \pm 0.2^{b}$ & $2.9 \pm 0.3^{b}$ & $0.2 \pm 0.0^{b}$ & $900 \pm 60^{b}$ & $12.4 \pm 0.3^{b}$ & $0.9 \pm 0.0^{b}$ & $0.1 \pm 0.0^{b}$ & $5.7 \pm 0.2^{b}$ & $2.4 \pm 0.2^{a}$ \\
\hline & Je3-3 & $5.2 \pm 0.1^{b}$ & $3.8 \pm 0.4^{b}$ & $0.3 \pm 0.0^{b}$ & $999 \pm 174^{b}$ & $11.6 \pm 0.4^{b}$ & $0.8 \pm 0.0^{b}$ & $0.1 \pm 0.0^{b}$ & $2.6 \pm 0.6^{b}$ & $0.6 \pm 0.2^{b}$ \\
\hline & Je4-1 & $6.7 \pm 0.3^{b}$ & $3.0 \pm 0.3^{b}$ & $0.2 \pm 0.0^{b}$ & $368 \pm 129^{c}$ & $9.2 \pm 0.6^{b}$ & $0.1 \pm 0.0^{c}$ & $0.1 \pm 0.0^{b}$ & $4.4 \pm 0.6^{b}$ & $1.4 \pm 0.3^{b}$ \\
\hline & Je4-2 & $7.7 \pm 0.2^{a}$ & $3.9 \pm 0.5^{b}$ & $0.3 \pm 0.0^{b}$ & $669 \pm 65^{b}$ & $9.3 \pm 0.1^{b}$ & $0.2 \pm 0.0^{b}$ & $0.1 \pm 0.0^{b}$ & $10.4 \pm 1.9^{a}$ & $0.8 \pm 0.1^{b}$ \\
\hline & Mean & $6.2 \pm 0.8$ & $2.9 \pm 1.0$ & $0.3 \pm 0.0$ & $737 \pm 271$ & $10.5 \pm 1.6$ & $0.6 \pm 0.3$ & $0.1 \pm 0.0$ & $4.8 \pm 2.3$ & $0.9 \pm 0.6$ \\
\hline \multirow{5}{*}{ Bonghwa } & Bon-1 & $6.1 \pm 0.4^{b}$ & $0.5 \pm 0.0^{c}$ & $0.1 \pm 0.0^{c}$ & $248 \pm 47^{c}$ & $5.4 \pm 0.4^{c}$ & $0.3 \pm 0.0^{b}$ & $0.0 \pm 0.0^{\mathrm{c}}$ & $4.0 \pm 0.1^{b}$ & $0.8 \pm 0.0^{b}$ \\
\hline & Bon-2 & $6.5 \pm 0.2^{b}$ & $1.5 \pm 0.1^{b}$ & $0.2 \pm 0.0^{b}$ & $415 \pm 33^{c}$ & $7.2 \pm 0.8^{b}$ & $0.5 \pm 0.1^{b}$ & $0.1 \pm 0.0^{b}$ & $3.3 \pm 0.1^{b}$ & $0.6 \pm 0.1^{b}$ \\
\hline & Bon-3 & $6.6 \pm 0.2^{b}$ & $3.2 \pm 0.2^{b}$ & $0.3 \pm 0.0^{b}$ & $165 \pm 50^{c}$ & $10.8 \pm 0.4^{b}$ & $0.9 \pm 0.1^{b}$ & $0.1 \pm 0.0^{b}$ & $5.1 \pm 0.3^{b}$ & $1.1 \pm 0.1^{b}$ \\
\hline & Bon-4 & $6.9 \pm 0.2^{b}$ & $1.5 \pm 0.2^{b}$ & $0.2 \pm 0.0^{b}$ & $953 \pm 9^{b}$ & $9.2 \pm 0.6^{b}$ & $0.2 \pm 0.0^{b}$ & $0.1 \pm 0.0^{b}$ & $5.4 \pm 0.1^{b}$ & $1.2 \pm 0.1^{b}$ \\
\hline & Mean & $6.4 \pm 0.4$ & $1.7 \pm 1.0$ & $0.2 \pm 0.0$ & $770 \pm 547$ & $7.8 \pm 2.1$ & $0.5 \pm 0.3$ & $0.1 \pm 0.0$ & $4.4 \pm 0.9$ & $0.9 \pm 0.2$ \\
\hline
\end{tabular}

* a, b, c: value in same columns with different superscripts are significantly different $(p<0.05)$ with one-way ANOVA and Duncan's test (alpha $=0.05)$ using SPSS statistics (IBM Corporation, v. 20.0 Armonk, NY, USA). 
Table 3. Growth characteristics of roots in Angelica gigas cultivation sites, Korea (mean \pm SD).

\begin{tabular}{|c|c|c|c|c|c|c|}
\hline Region & $\begin{array}{l}\text { Cultivation } \\
\text { Sites }\end{array}$ & $\begin{array}{l}\text { Fresh Weight of } \\
\text { Shoot (g) }\end{array}$ & $\begin{array}{l}\text { Fresh Weight of } \\
\text { Root (g) }\end{array}$ & $\begin{array}{l}\text { Dry Weight of } \\
\text { Root (g) }\end{array}$ & $\begin{array}{c}\text { Percent of Water Content } \\
\text { in Root }(\%)\end{array}$ & $\begin{array}{c}\text { Root/Shoot } \\
\text { Ratio }\end{array}$ \\
\hline \multirow{5}{*}{$\begin{array}{l}\text { Pyeong } \\
\text { chang }\end{array}$} & Pye1-1 & $243.8 \pm 201.2^{\mathrm{b}}$ & $495.2 \pm 259.4^{b}$ & $142.4 \pm 81.2^{b}$ & $70.4 \pm 12.0^{b}$ & $2.6 \pm 1.1^{b}$ \\
\hline & Pye1-2 & $169.0 \pm 102.0^{b}$ & $623.9 \pm 417.0^{a}$ & $167.9 \pm 138.5^{a}$ & $68.2 \pm 22.6^{b}$ & $3.8 \pm 1.4^{b}$ \\
\hline & Pye2-1 & $133.1 \pm 96.3^{b}$ & $374.7 \pm 207.3^{b}$ & $100.4 \pm 61.0^{\mathrm{b}}$ & $69.3 \pm 19.8^{b}$ & $3.8 \pm 2.5^{b}$ \\
\hline & Pye2-5 & $131.7 \pm 92.0^{b}$ & $541.3 \pm 285.8^{b}$ & $148.4 \pm 91.0^{\mathrm{b}}$ & $72.4 \pm 5.9^{b}$ & $5.3 \pm 2.5^{\mathrm{a}}$ \\
\hline & Mean & $176.6 \pm 151.5$ & $467.5 \pm 275.6$ & $130.1 \pm 86.9$ & $70.0 \pm 16.0$ & $3.6 \pm 2.2$ \\
\hline \multirow{11}{*}{ Jecheon } & Je1-1 & $140.8 \pm 84.7^{b}$ & $171.4 \pm 91.4^{\mathrm{c}}$ & $43.9 \pm 25.0^{b}$ & $72.2 \pm 6.0^{b}$ & $1.3 \pm 0.5^{c}$ \\
\hline & Je1-2 & $123.0 \pm 47.9^{b}$ & $270.0 \pm 166.4^{b}$ & $87.8 \pm 67.4^{b}$ & $69.4 \pm 3.9^{b}$ & $2.3 \pm 1.3^{b}$ \\
\hline & Je1-3 & $61.0 \pm 23.1^{c}$ & $131.0 \pm 62.4^{c}$ & $34.7 \pm 17.4^{c}$ & $73.4 \pm 3.2^{b}$ & $2.2 \pm 0.8^{b}$ \\
\hline & Je1-4 & $145.5 \pm 190.1^{b}$ & $250.5 \pm 106.6^{b}$ & $69.6 \pm 36.4^{b}$ & $73.1 \pm 3.1^{b}$ & $4.1 \pm 3.6^{b}$ \\
\hline & Je2-1 & $78.0 \pm 46.1^{b}$ & $201.0 \pm 158.5^{b}$ & $35.0 \pm 33.2^{c}$ & $79.1 \pm 6.8^{\mathrm{a}}$ & $3.1 \pm 2.8^{b}$ \\
\hline & Je3-1 & $179.8 \pm 89.7^{b}$ & $280.9 \pm 119.8^{b}$ & $69.8 \pm 32.8^{b}$ & $75.4 \pm 3.6^{b}$ & $1.8 \pm 0.8^{b}$ \\
\hline & Je3-2 & $217.7 \pm 122.7^{b}$ & $485.5 \pm 290.5^{b}$ & $149.4 \pm 94.7^{b}$ & $67.5 \pm 20.2^{b}$ & $2.5 \pm 1.5^{b}$ \\
\hline & Je3-3 & $168.6 \pm 117.6^{b}$ & $397.5 \pm 241.5^{b}$ & $115.5 \pm 85.6^{b}$ & $72.5 \pm 3.4^{b}$ & $2.7 \pm 1.6^{b}$ \\
\hline & Je4-1 & $110.5 \pm 28.2^{b}$ & $288.5 \pm 136.2^{b}$ & $84.3 \pm 42.9^{b}$ & $71.2 \pm 2.2^{b}$ & $2.8 \pm 1.7^{b}$ \\
\hline & Je4-2 & $70.3 \pm 85.8^{c}$ & $104.9 \pm 49.9^{c}$ & $33.5 \pm 19.1^{c}$ & $69.1 \pm 5.0^{b}$ & $2.3 \pm 1.0^{b}$ \\
\hline & Mean & $151.4 \pm 111.3$ & $293.9 \pm 218.6$ & $84.4 \pm 71.9$ & $71.9 \pm 9.7$ & $2.3 \pm 1.7$ \\
\hline \multirow{5}{*}{ Bonghwa } & Bon-1 & $213.3 \pm 72.0^{b}$ & $345.6 \pm 153.2^{b}$ & $106.6 \pm 48.8^{b}$ & $48.6 \pm 23.7^{c}$ & $1.7 \pm 0.7^{b}$ \\
\hline & Bon-2 & $124.4 \pm 76.0^{b}$ & $383.8 \pm 129.1^{b}$ & $96.2 \pm 28.3^{b}$ & $73.0 \pm 10.5^{b}$ & $5.1 \pm 3.8^{b}$ \\
\hline & Bon-3 & $283.0 \pm 163.5^{b}$ & $346.0 \pm 139.2^{b}$ & $107.8 \pm 59.9^{b}$ & $46.0 \pm 61.1^{b}$ & $1.7 \pm 1.3^{b}$ \\
\hline & Bon-4 & $315.0 \pm 186.7^{\mathrm{a}}$ & $361.1 \pm 144.8^{b}$ & $88.2 \pm 34.7^{b}$ & $68.5 \pm 8.5^{b}$ & $1.3 \pm 0.3^{c}$ \\
\hline & Mean & $238.3 \pm 154.2$ & $358.1 \pm 136.8$ & $100.0 \pm 44.4$ & $58.3 \pm 35.6$ & $2.3 \pm 2.5$ \\
\hline
\end{tabular}

a, b, c: value in same columns with different superscripts are significantly different $(p<0.05)$ with oneway. ANOVA and Duncan's test (alpha = 0.05) using SPSS statistics (IBM Corporation, v. 20.0 Armonk, NY, USA).

Table 4. Correlation coefficients between root growth characteristics and meteorological factors in Angelica gigas Nakai cultivation sites, Korea.

\begin{tabular}{cccc}
\hline Meteorological Factor & Dry Weight of Root & Percent of Water Content in Root (\%) & Root/Shoot Ratio \\
\hline Altitude $(\mathrm{m})$ & $0.646^{* *}$ & 0.187 & $0.608^{* *}$ \\
Mean temperature $\left({ }^{\circ} \mathrm{C}\right)$ & $-0.645^{* *}$ & -0.091 & $-0.498^{*}$ \\
Mean minimum temperature $\left({ }^{\circ} \mathrm{C}\right)$ & $-0.627^{* *}$ & -0.139 & -0.463 \\
Mean maximum temperature $\left({ }^{\circ} \mathrm{C}\right)$ & $-0.659^{* *}$ & -0.013 & $-0.497^{*}$ \\
The highest temperature $\left({ }^{\circ} \mathrm{C}\right)$ & $-0.604^{* *}$ & -0.185 & $-0.502^{*}$ \\
The lowest temperature $\left({ }^{\circ} \mathrm{C}\right)$ & $-0.650^{* *}$ & 0.093 & -0.412 \\
The mean temperature of dew point $\left({ }^{\circ} \mathrm{C}\right)$ & $-0.624^{* *}$ & -0.147 & $-0.503^{*}$ \\
Mean Air humidity $(\mathrm{g} / 100 \mathrm{~g})$ & $0.659^{* *}$ & 0.003 & $0.467^{*}$ \\
Annual precipitation $(\mathrm{mm})$ & -0.094 & $0.486^{*}$ & 0.142 \\
The duration of sunshine $(\mathrm{hr})$ & $0.547^{*}$ & 0.264 & $0.498^{*}$ \\
The percentage of sunshine $(\mathrm{g} / 100 \mathrm{~g})$ & $0.542^{*}$ & 0.269 & 0.482 \\
The minimum grass temperature $\left({ }^{\circ} \mathrm{C}\right)$ & $-0.613^{* *}$ & 0.192 & -0.354 \\
Dry weight of root & 1.000 & -0.112 & 0.132 \\
Percent of water content in root & -0.112 & -0.180 & -0.182 \\
Root/shoot ratio & 0.132 & -0.183 & 1 \\
\hline
\end{tabular}

*Significant at $p=0.05,{ }^{* *}$ Significant at $p=0.01$.

Table 5. Correlation coefficients between root growth characteristics and edaphic characteristics in Angelica gigas Nakai cultivation sites, Korea.

\begin{tabular}{cccc}
\hline Edaphic Characteristics & Dry Weight of Root & Percent of Water Content in Root & $\begin{array}{c}\text { Root/Shoot } \\
\text { Ratio }\end{array}$ \\
\hline $\mathrm{pH}$ & $-0.514^{*}$ & -0.185 & -0.34 \\
Gravel content & -0.282 & 0.254 & -0.232 \\
Organic matter & $0.590^{* *}$ & 0.376 & 0.39 \\
Total N & $0.506^{*}$ & 0.386 & 0.38 \\
Available $\mathrm{P}_{2} \mathrm{O}_{5}$ & 0.386 & 0.387 & 0.67 \\
$\mathrm{CEC}$ & $0.537^{*}$ & $0.568^{*}$ & 0.18 \\
The content of sand & 0.148 & 0.304 & 0.43 \\
The content of silt & -0.156 & -0.241 & -0.43 \\
The content of clay & -0.092 & -0.396 & -0.34 \\
$\mathrm{~K}^{+}$ & 0.037 & -0.017 & 0.00 \\
$\mathrm{Na}^{+}$ & $-0.560 *$ & -0.054 & 0.00 \\
$\mathrm{Ca}^{2+}$ & -0.239 & -0.111 & 0.20 \\
$\mathrm{Mg}^{2+}$ & 0.300 & 0.026 & 0.09 \\
\hline
\end{tabular}

*Significant at $p=0.05, * *$ Significant at $p=0.01$. 


\subsection{Characteristics of Active Compounds}

According to the analysis of active compounds from the four sites in Pyeongchang, nodakenin was at 0.29 to $0.35 \mathrm{~g} / 100 \mathrm{~g}$, decursin was at 2.90 to $3.22 \mathrm{~g} / 100 \mathrm{~g}$, and decursinolangelate was at 2.07 to $2.38 \mathrm{~g} / 100 \mathrm{~g}$, and the total of the three compounds was $5.63 \mathrm{~g} / 100 \mathrm{~g}$ on average (Table 6). The Jecheon area had 0.76 to $1.27 \mathrm{~g} / 100 \mathrm{~g}$ nodakenin, 2.49 to $4.42 \mathrm{~g} / 100 \mathrm{~g}$ decursin, and 2.03 to $3.43 \mathrm{~g} / 100 \mathrm{~g}$ decursinolangelate, which was higher than that of the Pyeongchang sites, and showed an average of $7.02 \mathrm{~g} / 100 \mathrm{~g}$ for the total of three components (Table 6). In the Bonghwa region, the value of nodakenin was 0.64 to $0.88 \mathrm{~g} / 100 \mathrm{~g}$, decursin 3.22 to $3.46 \mathrm{~g} / 100 \mathrm{~g}$, decursinolangelate 1.93 to $2.37 \mathrm{~g} / 100 \mathrm{~g}$, and the total content was $6.17 \mathrm{~g} / 100 \mathrm{~g}$ on average (Table 6). Thus, there was a difference in the content between regions, decreasing in value in the order of Jecheon, Bonghwa, and Pyeongchang, and overall, there was a tendency to have a high content of active compounds in sites with a low average value of root dry weight. Je1-3 in Jecheon had the lowest root dry weight among the 18 cultivation sites, but in terms of active compounds, statistically, the highest content was at $9.0 \mathrm{~g} / 100 \mathrm{~g}$ (Table 6, $p<0.05$ ). Among the active compounds, the nodakenin content was significantly low in the four sites in PyeongChang, while Je1-3, which had the highest total content, also had the highest levels of nodakenin content (Table 6, $p<0.05)$.

Table 6. Active compounds in root extracts of Angelica gigas Nakai cultivation sites, Korea (mean \pm SD).

\begin{tabular}{|c|c|c|c|c|c|}
\hline Region & Cultivation Sites & $\begin{array}{c}\text { Total Active } \\
\text { Compounds (g/100 g) }\end{array}$ & Nodakenin (g/100 g) & Decursin $(\mathrm{g} / 100 \mathrm{~g})$ & $\begin{array}{c}\text { Decursinol } \\
\text { Angelate }(\mathrm{g} / 100 \mathrm{~g})\end{array}$ \\
\hline \multirow{5}{*}{ Pyeongchang } & Pye1-1 & $5.27 \pm 1.31^{\mathrm{c}}$ & $0.30 \pm 0.09^{c}$ & $2.90 \pm 0.83^{b}$ & $2.07 \pm 0.57^{b}$ \\
\hline & Pye1-2 & $5.62 \pm 1.46^{b}$ & $0.29 \pm 0.07^{c}$ & $3.22 \pm 0.77^{b}$ & $2.11 \pm 0.68^{b}$ \\
\hline & Pye2-1 & $5.87 \pm 1.65^{b}$ & $0.35 \pm 0.09^{c}$ & $3.18 \pm 0.97^{b}$ & $2.34 \pm 0.68^{b}$ \\
\hline & Pye2-5 & $5.89 \pm 1.06^{b}$ & $0.29 \pm 0.10^{c}$ & $3.22 \pm 0.65^{b}$ & $2.38 \pm 0.50^{b}$ \\
\hline & Mean & $5.63 \pm 1.46$ & $0.31 \pm 0.10$ & $3.09 \pm 0.87$ & $2.22 \pm 0.63$ \\
\hline \multirow{11}{*}{ Jecheon } & Je1-1 & $5.28 \pm 1.38^{c}$ & $0.76 \pm 0.24^{b}$ & $2.49 \pm 0.70^{c}$ & $2.03 \pm 0.57^{b}$ \\
\hline & Je1-2 & $6.50 \pm 0.79^{b}$ & $0.89 \pm 0.21^{b}$ & $3.27 \pm 0.46^{b}$ & $2.34 \pm 0.31^{b}$ \\
\hline & Je1-3 & $9.03 \pm 2.33^{\mathrm{a}}$ & $1.18 \pm 0.29^{a}$ & $4.42 \pm 1.09^{\mathrm{a}}$ & $3.43 \pm 1.20^{a}$ \\
\hline & Je1-4 & $5.82 \pm 1.16^{b}$ & $0.89 \pm 0.14^{b}$ & $2.66 \pm 0.55^{b}$ & $2.26 \pm 0.57^{b}$ \\
\hline & Je2-1 & $7.07 \pm 1.73^{b}$ & $1.27 \pm 0.26^{\mathrm{a}}$ & $3.17 \pm 1.17^{b}$ & $2.63 \pm 0.71^{b}$ \\
\hline & Je3-1 & $7.61 \pm 1.80^{b}$ & $0.92 \pm 0.26^{b}$ & $4.12 \pm 0.91^{b}$ & $2.57 \pm 0.78^{b}$ \\
\hline & Je3-2 & $7.27 \pm 1.85^{b}$ & $0.95 \pm 0.27^{b}$ & $3.89 \pm 0.94^{b}$ & $2.43 \pm 0.87^{b}$ \\
\hline & Je3-3 & $7.84 \pm 2.14 b$ & $0.90 \pm 0.24^{b}$ & $3.90 \pm 1.14^{b}$ & $3.03 \pm 0.90^{b}$ \\
\hline & Je4-1 & $6.31 \pm 1.55^{b}$ & $1.20 \pm 0.38^{\mathrm{a}}$ & $2.79 \pm 0.79^{b}$ & $2.32 \pm 0.61^{b}$ \\
\hline & Je4-2 & $7.80 \pm 3.54^{b}$ & $1.01 \pm 0.55^{b}$ & $3.64 \pm 1.71^{b}$ & $3.15 \pm 1.34^{\mathrm{a}}$ \\
\hline & Mean & $7.02 \pm 2.18$ & $0.94 \pm 0.31$ & $3.50 \pm 1.71$ & $2.58 \pm 0.91$ \\
\hline \multirow{5}{*}{ Bonghwa } & Bon-1 & $6.24 \pm 1.64^{b}$ & $0.64 \pm 0.12^{b}$ & $3.22 \pm 0.82^{b}$ & $2.37 \pm 0.79^{b}$ \\
\hline & Bon-2 & $6.40 \pm 1.11^{b}$ & $0.88 \pm 0.28^{b}$ & $3.46 \pm 0.65^{b}$ & $2.06 \pm 0.39^{b}$ \\
\hline & Bon-3 & $6.14 \pm 1.31^{b}$ & $0.76 \pm 0.19^{b}$ & $3.27 \pm 1.01^{b}$ & $2.10 \pm 0.45^{b}$ \\
\hline & Bon-4 & $5.92 \pm 1.27^{b}$ & $0.75 \pm 0.11^{b}$ & $3.24 \pm 0.69^{b}$ & $1.93 \pm 0.64^{c}$ \\
\hline & Mean & $6.17 \pm 1.36$ & $0.76 \pm 0.20$ & $3.29 \pm 0.82$ & $2.12 \pm 0.61$ \\
\hline
\end{tabular}

a, b, c: value in same columns with different superscripts are significantly different $(p<0.05)$ with oneway ANOVA and Duncan's test (alpha =0.05) using SPSS statistics (IBMCorporation, Ver. 20.0 Armonk, NY, USA).

In addition, Je2-1, Je4-1 in the same Jecheon area showed significantly higher values of $1.20 \mathrm{~g} / 100 \mathrm{~g}$ to $1.27 \mathrm{~g} / 100 \mathrm{~g}$ of nodakenin content. The highest decursin content was in Je1-3 and the lowest was in Je1-1. Decursinolangelates, like the other two compounds, were statistically the highest in the Je1-3 and Je4-2 sites among the total 18 cultivation sites (Table 6,p<0.05). Bon-4 in the Bonghwa area had the lowest statistically significant levels of decursinolangelates among the entire cultivation sites.

Correlation analysis of the altitude, weather conditions, and the active compounds of the root extracts were carried out in Pyeongchang, Jecheon, and Bonghwa regions. It was found that the compound most affected by the weather conditions was nodakenin, which showed a positive correlation with temperature-related factors (Table 7 , significant at $p=0.01$ ). Nodakenin also showed a negative correlation with altitude, air humidity, root dry weight, the duration of sunshine, and the percentage of sunshine (Table 7 , significant at $p=0.01$ ). Decursin and ducursinolangelate did not show much relevance with the weather conditions. 
Table 7. Correlation coefficients between active compounds and meteorological factors in Angelica gigas Nakai cultivation sites, Korea.

\begin{tabular}{|c|c|c|c|c|}
\hline Meteorological Factors & $\begin{array}{l}\text { Total Content of Active } \\
\text { Compounds (g/100 g) }\end{array}$ & $\begin{array}{l}\text { Nodakenin } \\
(\mathrm{g} / 100 \mathrm{~g})\end{array}$ & $\begin{array}{l}\text { Decursin } \\
(\mathrm{g} / 100 \mathrm{~g})\end{array}$ & $\begin{array}{c}\text { Decursinol Angelate } \\
(\mathrm{g} / 100 \mathrm{~g})\end{array}$ \\
\hline Altitude & -0.403 & $-0.810^{* *}$ & -0.141 & -0.209 \\
\hline Mean temperature & $0.533 *$ & $0.896^{* *}$ & 0.242 & 0.341 \\
\hline Mean minimum temperature & 0.568 * & $0.911^{* *}$ & 0.250 & 0.408 \\
\hline Mean maximum temperature & $0.503 *$ & $0.875 * *$ & 0.233 & 0.293 \\
\hline The highest temperature & 0.468 & $0.845^{* *}$ & 0.223 & 0.244 \\
\hline The lowest temperature & $0.594^{* *}$ & $0.894^{* *}$ & 0.251 & $0.483 *$ \\
\hline The mean temperature of dew point & $0.497 *$ & $0.871^{* *}$ & 0.232 & 0.286 \\
\hline Air humidity & -0.574 * & $-0.912 * *$ & -0.251 & -0.421 \\
\hline Annual precipitation & 0.230 & 0.104 & 0.055 & 0.426 \\
\hline The duration of sunshine & -0.394 & $-0.769 * *$ & -0.197 & -0.148 \\
\hline The percentage of sunshine & -0.389 & $-0.763^{* *}$ & -0.196 & -0.141 \\
\hline The minimum grass temperature & $0.591 * *$ & $0.838^{* *}$ & 0.241 & 0.534 * \\
\hline Dry weight of root & -0.437 & $-0.727 * *$ & -0.076 & -0.439 \\
\hline Percent of water content in root & 0.126 & 0.160 & 0.106 & 0.067 \\
\hline Total content of active compounds & 1.000 & $0.659^{* *}$ & $0.874^{* *}$ & $0.905^{* *}$ \\
\hline Nodakenin & $0.659 * *$ & 1.000 & 0.310 & 0.494 * \\
\hline Decursin & $0.874^{* *}$ & 0.310 & 1.000 & $0.705^{* *}$ \\
\hline Decursinol angelate & $0.905^{* *}$ & 0.494 * & $0.705^{* *}$ & 1.000 \\
\hline
\end{tabular}

*Significant at $p=0.05,{ }^{* *}$ Significant at $p=0.01$.

In addition, the correlation between the soil characteristics of the sites and the content of active compounds was conducted and all three compounds showed a high degree of correlation with the content of the gravel (Table 8). Nodakenin also showed a positive correlation with soil $\mathrm{pH}$ and $\mathrm{Na}^{+}$ content (Table 8, significant at $p=0.01$ ). Decursinolangelate showed a positive correlation with $\mathrm{Ca}^{2+}$ content (Table 8 , significant at $p=0.05$ ).

Table 8. Correlation coefficients between active compounds and edaphic characteristics in Angelica gigas Nakai cultivation sites, Korea.

\begin{tabular}{|c|c|c|c|c|}
\hline Edaphic Characteristics & $\begin{array}{l}\text { Total Active Compounds } \\
\text { (g/100 g) }\end{array}$ & $\begin{array}{c}\text { Nodakenin } \\
(\mathrm{g} / 100 \mathrm{~g})\end{array}$ & $\begin{array}{l}\text { Decursin } \\
(\mathrm{g} / \mathbf{1 0 0} \mathrm{g})\end{array}$ & $\begin{array}{c}\text { Decursinol } \\
\text { Angelate }(\mathrm{g} / \mathbf{1 0 0} \mathrm{g})\end{array}$ \\
\hline $\mathrm{pH}$ & 0.455 & $0.744^{* *}$ & 0.232 & 0.278 \\
\hline Gravel content & $0.738^{* *}$ & $0.575^{*}$ & $0.631 *$ & $0.684^{* *}$ \\
\hline Organic matter & -0.166 & -0.563 * & 0.018 & 0.003 \\
\hline Total N & -0.123 & $-0.545^{*}$ & 0.034 & 0.068 \\
\hline Available $\mathrm{P}_{2} \mathrm{O}_{5}$ & -0.183 & -0.414 & 0.041 & -0.191 \\
\hline CEC & -0.010 & -0.356 & 0.191 & 0.014 \\
\hline The content of sand & -0.151 & -0.239 & -0.196 & 0.046 \\
\hline The content of silt & 0.117 & 0.241 & 0.142 & -0.067 \\
\hline The content of clay & 0.205 & 0.184 & 0.287 & 0.014 \\
\hline $\mathrm{K}^{+}$ & 0.133 & 0.073 & 0.289 & -0.082 \\
\hline $\mathrm{Na}^{+}$ & 0.430 & $0.714^{* *}$ & 0.140 & 0.350 \\
\hline $\mathrm{Ca}^{2+}$ & 0.400 & 0.199 & 0.239 & $0.550 *$ \\
\hline $\mathrm{Mg}^{2+}$ & 0.078 & 0.040 & 0.133 & 0.004 \\
\hline $\mathrm{EC}$ & -0.224 & -0.168 & -0.110 & -0.298 \\
\hline $\mathrm{NaCl}$ & -0.177 & -0.447 & -0.111 & 0.036 \\
\hline Dry weight of root & -0.437 & $-0.727^{* *}$ & -0.076 & -0.439 \\
\hline Percent of water content in root & 0.126 & 0.160 & 0.106 & 0.067 \\
\hline Total active compounds & 1.000 & $0.659^{* *}$ & $0.874 * *$ & $0.905^{* *}$ \\
\hline Nodakenin & $0.659 * *$ & 1.000 & 0.310 & 0.494 \\
\hline Decursin & $0.874^{* *}$ & 0.310 & 1.000 & $0.705^{* *}$ \\
\hline Decursinol angelate & $0.905^{* *}$ & $0.494 *$ & $0.705^{* *}$ & 1.000 \\
\hline
\end{tabular}

* Significant at $p=0.05,{ }^{* *}$ Significant at $p=0.01$.

\section{Discussion}

Generally, Korean Angelica grows naturally in high mountain areas and does not grow well in high-temperature areas. According to studies, areas with an accumulative temperature of less than $3700{ }^{\circ} \mathrm{C}$ are suitable for the cultivation site of Korean Angelica [22]. Growing Korean Angelica in low-altitude areas is not good for the growth due to high-temperature damage caused in summer. In particular, Korean Angelica has an advantage in growing in mountainous highlands where there is 
an average temperature between $20^{\circ} \mathrm{C}$ and $22^{\circ} \mathrm{C}$ in July and August [1]. In this study, Pyeongchang area showed a high average root mass per individual plant, which was $130.1 \mathrm{~g}$ under low-temperature long-day conditions, and in Jecheon (84.4 g) and Bonghwa (100.0 g), where the sites were located below a $500 \mathrm{~m}$ altitude, and showed a 23 and $35 \mathrm{~g} / 100 \mathrm{~g}$ decrease in root mass, respectively, compared to that of Pyeongchang area.

In the recent studies done in Korea, studies on the breeding and cultivation of medicinal plants have been actively conducted but little has been done for active compounds except for [23-25]. A study found that ginseng, a major medicinal crop in Korea, has a lower content of ginsenoside in better growth conditions [23]. In addition, due to different weather conditions during the growth period, ginseng grown in the vinyl house tended to have a higher weight of small roots and higher content for certain ginsenosides [24]. In conclusion, although growth was not good in the cultivation sites where the weather condition was not optimal due to a low altitude, the content of nodakenin was high.

Environmental factors, such as light conditions, soil acidity, moisture content, organic matter, nutritional saline content, etc., are very complex and such heterogeneity has a significant effect on plant growth and utility $[26,27]$. Although studies have been actively conducted on how the growth of herbs changes with these environmental factors [28-30], there have not been many studies on the properties of useful ingredients. The active compounds of plants are affected by a variety of factors, including weather, soil, and genetic factors, and each component is synthesized through different paths in different environments [31]. Foreign studies are being conducted to identify optimal reproductive conditions that could increase the specific functional component (index, active component) as well as the biomass of medicinal crops [32,33]. In addition, a medicinal component called the benzopheanthridine alkaloid, which has an antibacterial effect, had a good growth medium, but the component showed a high value in its natural habitat [34]. Furthermore, for medicinal plants native to Canada's maple forests, research has been published that favorable soil and light conditions for growth increase the total amount of useful ingredients [33]. Coumarin derivatives, including decursin and decursinolangelate, play an important role in the prevention of plant pathogens and in the response mechanism to non-biological [35]. In this study, a lot of gravel in the soil may have caused non-biological stress on this plant. As to the reasons for the above results, more detailed research is needed to pinpoint the factors that increase the content of each component. $\mathrm{Cu}$ ion is a well-known nonbiological elicitor and is known to be very effective in inducing the biosynthesis of cumarin compounds, which include decursin and decursinolangelate. The productivity of betacyanin, the same coumarin component, was increased by 1.3 times by the processing of $\mathrm{Cu}$ ions in the cell culture of Portulaca grandiflora and was reported to induce the production of coumarin compounds even in sunflowers [36]. In recent years, some studies have been conducted to increase the content of decursin and decursinolanegelate [37]. When agents induced with $\mathrm{Cu}$ ions were added to the yeast extract and were treated every two weeks during the first 10 weeks and then harvested, decursin and decursinolanegelate increased by about 1.5 to 1.7 times. In these papers, the technique of $\mathrm{Cu}$ ion treatment, how $\mathrm{Cu}$ ion is absorbed into the cells of the plants, and thus how active compounds are enhanced are explained [36,37].

\section{Materials and Methods}

\subsection{Study Sites}

This study was carried out in 4 to 10 cultivation sites according to the GAP (Great Agricultural Products Certification) cultivation patterns in Pyeongchang, Gangwon Province, Jecheon, Chungcheongbuk-do, and Bonghwa, Kyungsangbukdo Province (Table 9).

The location of each cultivation site was chosen as the study sites have little slope and are not obscured by surrounding trees. After raising seedlings for one year in the open field, they were then transplanted into the cultivation site. The 2-year-old seedlings of middle size, measuring 0.7 to 0.9 centimeters in root diameter, were planted in two rows at a 20-centimeter depth (interval of seedlings: between 25 and 30 centimeters). In Pyeongchang cultivation sites, except for the Pye2-5 
being at a 770-meter altitude, the remaining three cultivation sites were at a 674- to 697-meter altitude. In addition, 'Yeongheung' variety (No. 05-0032-5) registered in 2008 in the National Seed Resources Agency were transplanted starting from the end of March to early April. The cultivation sites of Jecheon is at 237 to 385-meter altitude. Except for Je1-1, Je3-2, and Je3-3, the seedlings were purchased from the seedling nursery site of 'Yeongheung' cultivar in PyeongChang. After a year of sowing seeds from Mt. Bangtae, the seedlings were then transplanted into the cultivation site in Je3-2 and Je3-3 (Table 9). The cultivation sites of Bonghwa were located at 318- to 378-meter altitude. Especially, Bon-2 was located in at the highest altitude among the four cultivation sites. The origin of seedlings in Bon-1, Bon-2, and Bon-3 was different since they were purchased from different places, and the seedlings of 'manchu' cultivar developed by the Rural Development Administration were planted in Bon-4 (Table 9).

Table 9. Angelica gigas Nakai cultivation sites on 2017 in Pyeongchang, Jecheon, and Bonghwa, Korea.

\begin{tabular}{|c|c|c|c|c|c|}
\hline Region & Province & Grower & Cultivation Sites & Origin & Altitude (m) \\
\hline \multirow{4}{*}{ Pyeongchang } & Sangjinbu-ri & A & Pye1-1 & Young Heung & 674 \\
\hline & Sangjinbu-ri & A & Pye1-2 & Young Heung & 674 \\
\hline & Tapdong-ri & B & Pye2-1 & Young Heung & 697 \\
\hline & Tapdong-ri & $\mathrm{C}$ & Pye2-5 & Young Heung & 770 \\
\hline \multirow{10}{*}{ Jecheon } & Wonwol-ri & $\mathrm{D}$ & Je1-1 & Bonghwa gun & 237 \\
\hline & Wonwol-ri & $\mathrm{E}$ & Je1-2 & Young Heung & 320 \\
\hline & Wonwol-ri & $\mathrm{F}$ & Je1-3 & Young Heung & 314 \\
\hline & Wonwol-ri & $\mathrm{F}$ & Je1-4 & Young Heung & 306 \\
\hline & Deokdong-ri & G & Je2-1 & Young Heung & 385 \\
\hline & Pojeon-ri & $\mathrm{H}$ & Je3-1 & - & 287 \\
\hline & Omi-ri & I & Je3-2 & Mt. Bangtae & 363 \\
\hline & Omi-ri & I & Je3-3 & Mt. Bangtae & 370 \\
\hline & Banghak-ri & $\mathrm{J}$ & Je4-1 & Young Heung & 288 \\
\hline & Wonwol-ri & $\mathrm{K}$ & Je4-2 & Young Heung & 299 \\
\hline \multirow{4}{*}{ Bonghwa } & Buncheon-ri & M & Bon-1 & - & 334 \\
\hline & Buncheon-ri & $\mathrm{N}$ & Bon-2 & - & 378 \\
\hline & Hyeondong-ri & $\mathrm{O}$ & Bon-3 & - & 318 \\
\hline & Hyeondong-ri & M & Bon-4 & Manchu & 320 \\
\hline
\end{tabular}

\subsection{Soil Analysis}

Soil cores within the root zone of the plant were sampled at three locations in the cultivation sites. The soil analysis was commissioned to the Korea Forestry Promotion Institute for all the necessary tests except for the gravel content. The content of gravel was measured by the weight of a gravel component of $2 \mathrm{~mm}$ or more when the specimen was dried and sifted using a sieve of $2 \mathrm{~mm}$. The chemical properties of the soil were measured with a specimen that passed through a 2-mm sieve. Soil texture analysis, which can measure the content of sand, mass, and clay in soil, was measured based on Stokes's law [38]. The organic content in the soil was measured by the weight difference of soil organic matter (g/100 g) after burning the organic matter in the soil immediately after drying at $600{ }^{\circ} \mathrm{C}$ for six hours (the soil organic matter content $(\mathrm{g} / 100 \mathrm{~g})=[($ WBefore - Wafter $) /$ WBefore $\times 100)$. Soil $\mathrm{pH}$ was measured using a $\mathrm{pH}$ meter and the soil samples were mixed with distilled water at a ratio of 1:5. The nitrogen content was measured using the Micro Kjeldahl Act [39], the Bray No.1 [40], and the cation exchange capacity [41]. The cation content of $\mathrm{Ca}, \mathrm{K}, \mathrm{Mg}$, and $\mathrm{Na}$ was measured using the plasma emission photometric method [41].

\subsection{Meteorological Data}

Data on weather conditions at the cultivation sites were collected from Daegwallyeong, Jecheon and Bonghwa branches, using the Korean Meteorological Administration's open portal (www.data.kma. go.kr). In addition, the atmospheric temperature and moisture systems (S-TMB-M002, ONset Computer Corporation, Bourne, MA, USA) and soil temperature and humidity measuring devices (S-TMB-002, S-SMA-005M-On) were installed on the two to three cultivation sites from July to September, 2017. 
However, the Pye2-5 was not included in the analysis as data was unavailable since September 6, 2017. Bonghwa area was evaluated from September 21 to November 2, 2017. According to the data from the Korea Meteorological Administration's Daegwallyeong branch, the monthly mean temperature of Pyeongchang from April to October 2017, which is the period of the growth of Korean Angelica, was $14.7^{\circ} \mathrm{C}$, which is 3.2 to $3.8^{\circ} \mathrm{C}$ lower than that of the Jecheon and Bonghwa regions (Table 10).

Table 10. Meteorological characteristics of Angelica gigas Nakai cultivation sites (data measured in Korea Meteorological Administration from April to October 2017).

\begin{tabular}{cccc}
\hline Meteorological Characteristics & Pyeongchang & Jecheon & Bonghwa \\
\hline Mean temperature $\left({ }^{\circ} \mathrm{C}\right)$ & 14.7 & 18.5 & 17.9 \\
The highest temperature $\left({ }^{\circ} \mathrm{C}\right)$ & 31.1 (July) & 36.8 (August) & 34.6 (August) \\
The lowest temperature $\left({ }^{\circ} \mathrm{C}\right)$ & -5.3 (October) & -5.1 (October) & -5.1 (October) \\
Accumulative temperature $\left({ }^{\circ} \mathrm{C}\right)$ & 3123.3 & 3959.5 & 3810.3 \\
Mean dew point temperature $\left({ }^{\circ} \mathrm{C}\right)$ & 6.1 & 7.5 & 8.1 \\
Mean relatively humidity $(\mathrm{g} / 100 \mathrm{~g})$ & 85.0 & 74.0 & 78.0 \\
Mean total precipitation $(\mathrm{mm})$ & 937.0 & 948.6 & 725.2 \\
Sum of sunshine duration $(\mathrm{h})$ & 1708.6 & 1355.9 & 1271.2 \\
Mean percentage of sunshine $(\mathrm{g} / 100 \mathrm{~g})$ & 59.7 & 47.6 & 44.5 \\
\hline
\end{tabular}

Furthermore, data showed the months with the highest temperature in the three cultivation sites. Pyeongchang had $31.1^{\circ} \mathrm{C}$ in July, while in August, Jecheon and Bonghwa had 36.8 and $34.6^{\circ} \mathrm{C}$ respectively (Table 10). The lowest temperature readings were $-5.1^{\circ} \mathrm{C}$ in October for both Bonghwa and Jecheon while it reached $-5.3^{\circ} \mathrm{C}$ in Pyeongchang (Table 10). The accumulative temperature based on $5^{\circ} \mathrm{C}$ was $3123.3^{\circ} \mathrm{C}$ for Pyeongchang, the lowest among the three regions, and Jecheon had the highest temperature of $3959.5^{\circ} \mathrm{C}$ (Table 10). The average dew point temperature was $6.1^{\circ} \mathrm{C}$ for Pyeongchang, the lowest among the three regions, and $8.1^{\circ} \mathrm{C}$ for Bonghwa (Table 10). The monthly mean relative humidity was $85.0 \%$, the highest among the three regions in Pyeongchang, and the lowest in Jecheon at $74.0 \%$. The total precipitation was the highest at $948.6 \mathrm{~mm}$ in Jecheon, and the lowest at $725.2 \mathrm{~mm}$ in Bonghwa (Table 10). In contrast to the temperature, Pyeongchang had the longest duration of sunshine exposure, with a mean percentage of $59.7 \%$ (Table 10 ).

\subsection{Analysis of the Growth Characteristics}

During the optimum harvest time, between 10 and 36 of the samples were collected from four cultivation sites in Pyeongchang, 10 cultivation sites in Jecheon, and 4 cultivation sites in Bonghwa between October 11 and 13. After the fresh weight of the root and the shoot were measured, they were dried at 35 to $40{ }^{\circ} \mathrm{C}$ for 10 days until there was no change in weight in the method that growers use and then the final weight for the dry one was measured.

\subsection{Analysis of Active Compounds}

Each sample was labeled and then crushed, and $10 \mathrm{mg}$ was extracted using $1 \mathrm{~mL}$ of $100 \% \mathrm{MeOH}$ for one hour. Extracts were centrifuged for $20 \mathrm{~min}$ at $3000 \mathrm{rpm}$ and filtered with a $0.2-\mu \mathrm{m}$ membrane filter (Whatman PTFE Syringe Filter, UK) for analysis. The content analysis was conducted using decursin, decursinolangelate, and nodakenin, which are the indicator components of the Korean Angelica as standard substances purchased from the National Institute for Korean Medicine Development and had a purity of $97 \%$ or more. $\mathrm{MeOH}$, acetonitrile, formic acid, and water, used in extraction and UPLC analysis, was purchased from J. T. Baker (USA) products, and formic acid used was from Sigma-Aldrich (USA) products. Analysis of the standard substances and extracts of samples were analyzed using the Waters Acuity I-class UPLC system (Waters, USA), and the HPLC conditions shown in the Korean Pharmaceuticals were modified to establish the optimal analysis method for UPLC conditions. The column used was the Waters Acquity BEH C18 column $(1.7 \mu \mathrm{m}, 2.1 \times 100 \mathrm{~mm})$ and the temperature of the column was maintained at $35^{\circ} \mathrm{C}$. The mobile phase started with a ratio of solution $\mathrm{A}$ $(0.1 \%$ formic acid in water): Solution B (acetonitrile containing $0.1 \%$ formic acid). The ratio was started 
to $80: 20 \rightarrow 1 \mathrm{~min}, 70: 30 \rightarrow 3 \mathrm{~min}, 50: 50 \rightarrow 3.5 \mathrm{~min}, 45: 55 \rightarrow 9 \mathrm{~min}, 0: 100 \rightarrow 9.5 \mathrm{~min}, 0: 100 \rightarrow 10.5 \mathrm{~min}$, and was finished to $80: 20 \rightarrow 12 \mathrm{~min}$. The sample injection volume was $2 \mu \mathrm{L}$, and the PDA detector was measured at UV $330 \mathrm{~nm}$. Based on the analysis conditions established, the three compounds nodakenin, decursin, and decursinolangelate were analyzed for six concentrations $(200,100,50,25$, 12.5, and $6.25 \mu \mathrm{g} / \mathrm{mL}$ ) and each calibration curve was prepared. The calibration coefficient value of the calibration curve was determined, and the contents of each compound were calculated (Table 11, $\left.\mathrm{r}^{2} \geq 0.997\right)$. The peak width of each compound was measured by analyzing each chromatogram obtained through the above analysis method through Empower Software 2.0. The measured width was calculated in $\mathrm{mg} / \mathrm{mL}$ using the expression of the calibration curve (Figure 1).

Table 11. Calibration curve equations of Nodakenin, Decursin, and Decursinol angelate.

\begin{tabular}{ccc}
\hline Compounds & Calibration Curve Equation & $\mathbf{r}^{2}$ \\
\hline Nodakenin & $\mathrm{y}=26063 \mathrm{x}+5418$ & 0.999 \\
\hline Decursin & $\mathrm{y}=34383 \mathrm{x}+65687$ & 0.999 \\
Decursinol angelate & $\mathrm{y}=29111 \mathrm{x}+25040$ & 0.999 \\
\hline
\end{tabular}

$\mathrm{Y}$ and $\mathrm{x}$ are the peak area and the concentration of the analytes respectively.

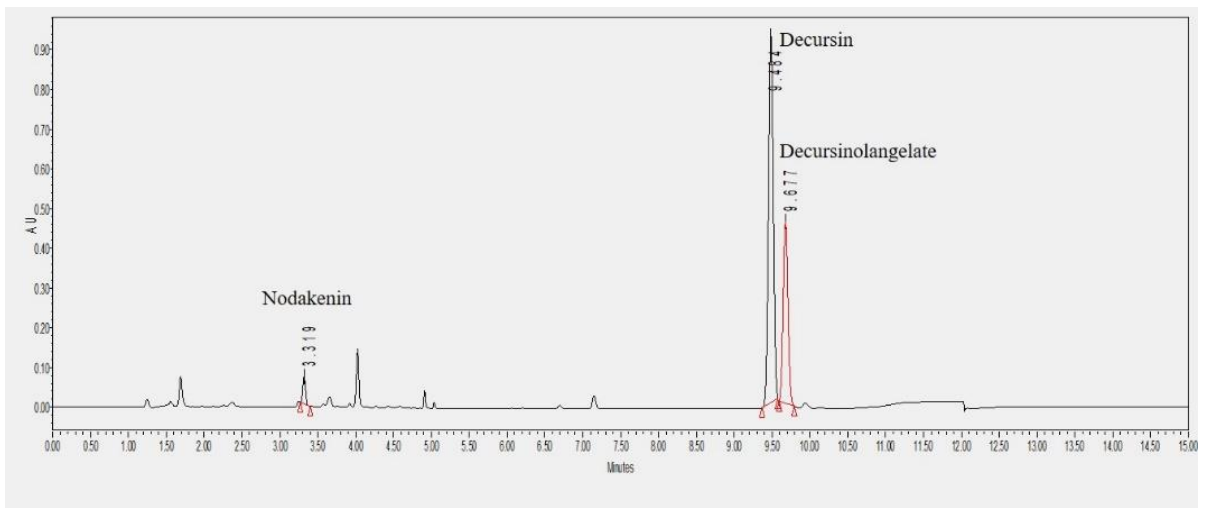

Figure 1. UPLC chromatogram of indicator components for the Angelica gigas samples.

\subsection{Data Analysis}

The soil and growth characteristics of the cultivation sites were not divided into regions, and MANOVA was carried out using the SPSS 20.0 Program (IBM Corporation, v. 20.0 Armonk, NY, USA) to determine if the total 18 cultivation sites in Pyeongchang, Jecheon, and Bonghwa showed statistically significant differences, and the follow-up test Duncan's test was used. The active compounds were averaged using the data analyzed three times per specimen of an object, and statistical processing was performed in a manner similar to the evaluation of the plant growth characteristics. A correlation analysis was performed to identify the link between the environmental characteristics, the plant growth characteristics, and active compounds, and the Pearson correlation coefficient was used for the analysis (SPSS v. 20.0).

\section{Conclusions}

The root dry weight from the cultivation site increased significantly when the conditions of the environment were similar to the natural habitat. Pyeongchang, which has a high root weight, was located in a $674 \sim 770 \mathrm{~m}$ altitude with an accumulative temperature of $3123^{\circ} \mathrm{C}$ and an organic matter content of $6.9 \pm 1.9 \%$, total $\mathrm{N}$ content of $0.4 \pm 0 \%, 1121.3 \pm 382.0 \mathrm{mg}^{-1} \mathrm{~kg}^{-1}$ of available $\mathrm{P}_{2} \mathrm{O}_{5}$ content, and CEC of $14.81 \pm 2.6 \mathrm{cmolc} \mathrm{kg}^{-1}$. Correlation analysis of active compounds showed that decursin was high in groups with a high content of soil gravel. Decursinolangelates showed the highest correlation with the content of soil gravel in the site, while nodakenin was high in the environments with high annual average temperatures and low solar hours, which was during the time period of April to 
October. Currently, cultivation sites show less than $6.0 \mathrm{~g} / 100 \mathrm{~g}$ of active compounds due to the weather conditions of the year and the environmental condition of the site; therefore, the domestic production of Korean Angelica is often not used as a raw material for functional food. This study concludes that the root yield in the cultivation sites would improve if weather conditions are maintained at a similar level as in their natural habitat. Additionally, the environment that improves root growth did not increase the content of active compounds, and when there was a high gravel content and high temperature during the growth period, the content of active compounds increased.

Author Contributions: Involved in every stage of this study, manuscript writing, and data analysis, Y.P.; Conducted in field survey of A. gigas, D.H.J., H.P., K.S.J., and Y.U.; Conducted the UPLC experiment, S.S. and N.K.; Collected A. gigas samples and carried out field productivity test, technically designed and helped inwriting the manuscript, P.S.P. and M.J.-K.; All authors have read and agreed to the published version of the manuscript.

Funding: This study was supported by the National Institute of Forest Science, Korea (project number FG0502-2017-01), and by the National Research Foundation of Korea (NRF) funded by Korea government (NRF-2017R1A2C1087099)

Acknowledgments: The authors extend their appreciation to the funding sources that enabled the research work to be carried out.

Conflicts of Interest: The authors declare that they have no conflict of interest.

\section{References}

1. Ahn, S.D.; Yu, C.Y.; Seo, J.S. Effect of temperature and daylength on growth and bolting of Angelica gigas Nakai. Korean J. Med. Crop Sci. 1994, 2, 20-25.

2. Choo, B.K.; Ji, Y.; Moon, B.C.; Lee, A.; Chun, J.M.; Yoon, T.; Kim, H.K. A study on environment characteristics of Angelica gigas Nakai Population. J. Korean Environ. Res. Technol. 2009, 12, 92-100.

3. Park, Y.; Jeong, D.; Sim, S.; Kim, N.; Park, H.; Jeon, G.S. The Characteristics of Growth and Active Compounds of Angelica gigas Nakai Population in Mt. Jeombong. Korean J. Plant Res. 2019, 32, 9-18.

4. Wink, M.; Schimmer, O. Modes of action of defensive secondary metabolites. In Functions of Plant Secondary Metabolites and Their Exploitation in Biotechnology; Wink, M., Ed.; CRC Press: Boca Raton, FL, USA, 1999.

5. Kaufman, P.B.; Cseke, L.J.; Warber, S.; Duke, J.A.; Brielmann, H.L. Natural Products from Plants; CRC Press: Boca Raton, FL, USA, 1999.

6. Briskin, D.P. Medicinal plants and phytomedicines: Linking plant biochemistry and physiology to human health. Plant Physiol. 2000, 124, 507-514. [CrossRef] [PubMed]

7. Kim, S.A.; Oh, H.K.; Kim, J.Y.; Hong, J.W.; Cho, S.I. A review of pharmacological effects of Angelica gigas, Angelica sinensis, Angelica acutiloba and their bioactive compounds. J. Korean Orient. Med. 2011, 32, 1-24.

8. Yu, H.S.; Park, C.H.; Park, C.G.; Kim, Y.G.; Park, H.W.; Seong, N.S. Growth characteristics and yield of the three species of genus Angelica. Korean J. Med. Crop Sci. 2004, 12, $43-46$.

9. Mao, X.; Kong, L.; Luo, Q.; Li, X.; Zou, H. Screening and analysis of permeable compounds in radix Angelica sinensis with immobilized liposome chromatography. J. Chromatogr. B 2002, 779, 331-339. [CrossRef]

10. Du, L.; Wang, X.; Cai, C.; Wang, T. Constituent analysis of essential oils from Radix of Angelica acutiloba. Zhong Yao Cai 2002, 25, 477-478.

11. Lu, G.H.; Chan, K.; Chan, C.L.; Leung, K.; Jiang, Z.H.; Zhao, Z.Z. Quantification of ligustilides in the roots of Angelica sinensis and related umbelliferous medicinal plants by high performance liquid chromatography and liquid chromatography-mass spectrometry. J. Chromatogr. B 2004, 1046, 101-107. [CrossRef]

12. Song, G.Y.; Lee, J.H.; Cho, M.; Park, B.S.; Kim, D.E.; Oh, S. Decursin suppresses human androgenin dependent PC3 prostate cancer cell proliferation by promoting the degradation of betacatenin. Mol. Pharmacol. 2007, 72, 1599-1606. [CrossRef]

13. Cho, N.J.; Lee, W.H.; Kim, K.K.; Han, H.S. Investigation of antioxidant effect of Angelicae Radix from Korea, China and Japan. J. Physiol. Pathol. Korean Med. 2017, 31, 182-187. [CrossRef]

14. Lam, H.W.; Lin, H.C.; Lao, S.C.; Gao, J.L.; Hong, S.J.; Leong, C.W. The angiogenic effects of Angelica sinensis extract on HUVEC in vitro and zebrafish in vivo. J. Cell. Biochem. 2008, 103, 195-211. [CrossRef] [PubMed]

15. Lee, S.; Shin, D.S.; Kim, J.S.; Oh, K.B.; Kang, S.S. Antibacterial coumarins from Angelica gigas roots. Arch. Pharm. Res. 2003, 26, 449-452. [CrossRef] [PubMed] 
16. Rhyu, M.R.; Kim, J.H.; Kim, E.Y. Radix Angelica elicits both nitric oxide-dependent and calcium influx-mediated relaxation in rat aorta. J. Cardiovas. Pharmacol. 2005, 46, 99-104. [CrossRef]

17. Kang, S.Y.; Lee, K.Y.; Sung, S.H.; Park, M.J.; Kim, Y.C. Coumarins isolated from Angelica gigas inhibit acetylcholinesterase: Structure-activity relationships. J. Nat. Prod. 2001, 64, 683-685. [CrossRef]

18. Kang, S.Y.; Kim, Y.C. Decursinol and decursin protect primary cultured rat cortical cells from glutamate-induced neurotoxicity. J. Pharm. Pharmacol. 2007, 59, 863-870. [CrossRef]

19. Ahn, K.S.; Sim, W.S.; Kim, I.H. Decursin: A cytotoxic agent and protein kinase C activator from the root of Angelica gigas. Planta Med. 1996, 62, 7-9. [CrossRef]

20. Shin, S.; Jeon, J.H.; Park, D.; Jang, J.Y.; Joo, S.S.; Hwang, B.Y.; Choe, S.Y.; Kim, Y.B. Anti-inflammatory effects of an ethanol extract of Angelica gigas in a carrageenan-air pouch inflammation model. Exp. Anim. 2009, 58, 431-436. [CrossRef]

21. Rural Development Administration. 2017. Available online: www.agdcm.kr (accessed on 8 June 2020).

22. Kim, Y.G.; Ahn, Y.S.; Ahn, T.J.; Yeo, J.H.; Park, C.B.; Park, H.K. Effect of yield and decursin content according to the accumulative temperature and seedling size in cultivation areas of Angelica gigas Nakai. Korean J. Med. Crop Sci. 2009, 17, 458-463.

23. Lee, M.K.; Park, H.; Lee, C.H. Effect of growth conditions on saponin content and ginsenoside pattern of Panax ginseng. Korea-Japan Panax Ginseng Symp. 1987, 9, 89-107.

24. Lee, S.W.; Kim, G.S.; Hyun, D.Y.; Kim, Y.B.; Kim, J.W.; Kang, S.W.; Cha, S.W. Comparison of growth characteristics and ginsenoside content of ginseng (Panax ginseng C. A. Meyer) cultivated with greenhouse and traditional shade facility. Korean J. Med. Crop Sci. 2011, 19, 157-161. [CrossRef]

25. Suh, J.T.; Cho, E.Y.; Yoo, D.L.; Kim, K.D.; Lee, J.N.; Hong, S.Y.; Kim, S.J.; Nam, J.H.; Han, H.M.; Kim, M.J. Comparative study of biological activities at different harvesting times and new varieties for highland culture of gom-chwi. Korean J. Plant Res. 2015, 28, 391-399. [CrossRef]

26. Koch, A.S.; Matzner, E. Heterogeneity of soil and soil solution chemistry under Norway spruce (Picea abies Karst.) and European beech (Fagus sylvatica L.) as influenced by distance from the stem basis. Plant soil. 1993, 151, 227-237. [CrossRef]

27. Robertson, G.P.; Huston, M.A.; Evans, F.C.; Tiedje, J.M. Spatial variability in a successional plant community: Patterns of nitrogen availability. Ecology 1988, 69, 1517-1524. [CrossRef]

28. Kephart, S.R.; Paladino, C. Demographic change and microhabitat variability in a grassland endemic, Silene douglasii var. oraria (Caryophyllaceae). Am. J. Bot. 1997, 84, 179-189. [CrossRef] [PubMed]

29. Munzbergova, $Z$. Determinants of species rarity; population growth rates of species sharing the same habitat. Am. J. Bot. 2005, 2, 1987-1994. [CrossRef]

30. Vellend, M. Land-use history and plant performance in populations of Trillium grandiflorum. Biol. Conserv. 2005, 124, 217-224. [CrossRef]

31. Bourgaud, F.; Hehn, A.; Larbat, R.; Doerper, S.; Gontier, E.; Kellner, S.; Matern, U. Biosynthesis of coumarins in plants: A major pathway still to be unravelled for cytochrome P450 enzymes. Phytochem. Rev. 2006, 5, 293-308. [CrossRef]

32. Fournier, A.R.; Proctorb, J.T.A.; Gauthierc, L.; Khanizadehd, S.; Be'langerd, A.; Gosselina, A.; Doraise, M. Understory light and root ginsenosides in forest-grown Panax quinquefolius. Phytochemistry 2003, 63, 777-782. [CrossRef]

33. Naud, J.; Olivier, A.; Be'langer, A.; Lapointe, L. Medicinal understory herbaceous species cultivated under different light and soil conditions in maple forests in southern Que'bec, Canada. Agroforest Syst. 2010, 79, 303-326. [CrossRef]

34. Graf, T.N.; Levine, K.E.; Andrews, M.E.; Perlmutter, J.M.; Nielsen, S.J.; Davis, J.M.; Wani, M.C.; Oberlies, N.H. Variability in the yield of benzophenanthridine alkaloids in wildcrafted vs cultivated Bloodroot (Sanguinaria canadensis L.). J. Agric. Food Chem. 2007, 55, 1205-1211. [CrossRef]

35. Brown, S.A. Coumarins. In The Biochemistry of Plants-A Comprehensive Treatise; Stumpf, P.K., Conn, E.E., Eds.; Academic Press: New York, NY, USA, 1981; Volume 7, pp. 269-300.

36. Gutiérrez-Coronado, M.A.; Parry, A.; Tena, M.; Jorrin, J.; Edwards, R. Abiotic elicitation of coumarin phytoalexins in sunflower. Phytochemistry 1995, 38, 1185-1191. [CrossRef]

37. Rhee, H.S.; Cho, H.Y.; Son, S.Y.; Yoon, S.H.; Park, J.M. Enhanced accumulation of decursin and decursinol angelate in root cultures and intact roots of Angelica gigas Nakai following elicitation. Plant Cell Tissue Organ Cult. 2010, 101, 295-302. [CrossRef] 
38. Sparks, D.L. Environmental Soil Chemistry; Academic Press: San Diego, CA, USA, 1995.

39. Konen, M.E.; Jacobs, P.M.; Burras, C.L.; Talaga, B.J.; Mason, J.A. Equations for predicting soil organic carbon using loss-on-ignition for north central U.S. Soil Science. Soil Sci. Soc. Am. J. 2002, 66, 1878-1881. [CrossRef]

40. Kuo, S. Phosphorus. In Methods of Soil Analysis, Part 3: Chemical Methods; Sparks, D.L., Ed.; Soil Science Society of America Book Series Number 5; American Society of Agronomy: Madison, WI, USA, 1996; p. 1390.

41. Sumner, M.E.; Miller, W.P. Cation exchange capacity and exchange coefficients. In Methods of Soil Analysis. Part 3: Chemical Methods; Sparks, D.L., Ed.; Soil Science Society of America Book Series No. 5, 3rd ed. Soil Science Society of America: Madison. WI, USA, 1996; pp. 1201-1229.

(C) 2020 by the authors. Licensee MDPI, Basel, Switzerland. This article is an open access article distributed under the terms and conditions of the Creative Commons Attribution (CC BY) license (http://creativecommons.org/licenses/by/4.0/). 